\title{
Does adiponectin play a role in the pathogenesis of chronic spontaneous urticaria?
}

\author{
KATARZYNA ADAMCZYK', DOMINIKA WCISLO-DZIADECKAㄹ, MARTYNA ZBICIAK-NYLEC ${ }^{l}$, \\ LIGIA BRZEZINSKA-WCISLO ${ }^{1}$,ZENON BRZOZA ${ }^{3,4}$
}

${ }^{1}$ Department of Dermatology, School of Medicine in Katowice, Medical University of Silesia, Poland

${ }^{2}$ Department of Skin Structural Studies, Chair of Cosmetology, School of Pharmacy with Division of Laboratory Medicine in Sosnowiec, Medical University of Silesia, Poland

${ }^{3}$ Department of Internal Diseases, Allergology and Clinical Immunology, School of Medicine with Division of Dentistry in Zabrze, Medical University of Silesia, Poland

${ }^{4}$ Department of Internal Diseases with Division of Allergology, Institute of Medical Sciences, University of Opole, Poland

\begin{abstract}
Introduction: Chronic spontaneous urticaria constitutes an interdisciplinary problem and its pathogenesis is still a subject of debate. Overweight and hyperlipidemia are supposed to be related to chronic spontaneous urticaria. Fatty tissue can be the source of adipokines.

Aim of the study: To assess the potential role of adiponectin in chronic spontaneous urticaria pathogenesis.

Material and methods: The study included 52 chronic spontaneous urticaria patients and 43 healthy controls. The patients were divided into two subgroups: patients with wheals only, and patients with urticaria and an accompanying angioedema. The adiponectin concentration was measured in all studied subjects.

Results: No statistically significant difference in adiponectin level was determined between the studied groups and subgroups.

Conclusions: We are among the first to present the results of study to determine a possible role of adipokines in chronic spontaneous urticaria pathogenesis. We did not observe any difference in adiponectin level. In our opinion, it is necessary to conduct further analyses in this field.
\end{abstract}

Key words: adiponectin, adipokines, chronic urticaria, metabolism, pathogenesis.

(Centr Eur J Immunol 2020; 45 (1): 56-59)

\section{Introduction}

Urticaria constitutes a heterogeneous group of diseases characterized by the occurrence of urticarial wheals, which may be associated with angioedema symptoms. According to its definition, chronic urticaria involves wheals recurring over 6 weeks [1]. Chronic spontaneous urticaria (CSU) is a disease with complex etiology, which cannot be determined in many cases. In recent years, numerous studies have been conducted in order to evaluate pathomechanisms causing the disease. Among potential factors that may play a role in CSU pathogenesis, one may mention autoimmune processes, chronic infections, pseudoallergies, and presence of a chronic inflammatory condition, which is observed, among others, in obesity [2-4].

Fat tissue constitutes a source of biologically active substances - adipokines, which - thanks to their proinflammatory action - may activate mast cells, leading to wheal development. One of the main hormones in the fat tissue is adiponectin, a protein with proven anti-atherosclerotic, anti-diabetic, and anti-inflammatory effects in the course of metabolic and cardiovascular diseases [5]. Studies of recent years suggest an unfavorable, pro-inflammatory role of this hormone in the development of autoimmunological diseases [6]. The effect of adipokines on the development and course of CSU has not been determined so far. In this work, the authors made an effort to assess the potential relationship between adiponectin concentration and CSU.

\section{Material and methods}

The study included $52 \mathrm{CSU}$ patients (34 women and 18 men; average age, $40.63 \pm 12.39$ ) (TotU). The patients were divided into two subgroups: 25 patients with wheals as the only visible symptoms (U), and 27 patients with angioedema symptoms apart from wheals (U-Ae). The disease was diagnosed on the basis of whole clinical picture, excluding coexisting diseases. The urticaria activity score (UAS) seven-day monitoring was used to assess dis-

Correspondence: Zenon Brzoza, MD, PhD, Department of Internal Diseases with Division of Allergology, Institute of Medical Sciences, University of Opole, Poland, e-mail: zbrzoza@mp.pl Submitted: 22.05.2018; Accepted: 26.10.2018 
ease intensity. This questionnaire calculates the number of wheals and the intensity of pruritus. The control group was composed of 43 healthy volunteers (29 women and 14 men; average age, $39.21 \pm 11.57)$. The adiponectin concentration was determined in blood serum (R\&D), and body mass index (BMI) was calculated for the whole group and each subgroup.

Mann-Whitney U-test was used in statistical calculations. The study was approved by the Bioethics Committee of the Medical University of Silesia in Katowice, Poland. All subjects provided written consent.

\section{Results}

The comparison of the whole tested group with the control group did not show any statistically important difference in adiponectin concentration (Table 1). Similarly, no statistically important differences were found in adiponectin concentration, when comparing the two subgroups of the study group and controls (Table 1, Fig. 1). Additionally, no differences were observed in BMI and UAS values (Table 1).

\section{Discussion}

Among potential factors that participate in the CSU pathogenesis, one may mention the presence of a chronic inflammatory condition observed, among others, in obese patients, particularly in a group with concurrent metabolic syndrome [7]. Epidemiologic studies of Italian population showed obesity as more frequently occurring in CSU patients, whereas results of Polish studies show a relationship between overweight and obesity and CSU occurrence and durability of CSU symptoms [8, 9]. Moreover, the frequency of metabolic syndrome occurrence is significantly higher in CSU patients comparing with healthy people [10]. The inflammatory process in the course of obesity manifests itself with an increased level of concentration of pro-inflammatory cytokines, including TNF- $\alpha$, IL-6, and CRP [5].

Fat tissue constitutes a source of biologically active substances called "adipokines", which play multidirectional functions in human body and include cytokines, growth fac- tors, and hormones. In literature regarding this subject, many works emphasizing the relationship between adipokines and the development of type 2 diabetes, atherosclerosis, and metabolic syndrome can be found. However, the role of adipokines in CSU pathogenesis has not been fully explained. Determination of the relationship between adipokines and CSU seems to be of particular importance in the view of advancing obesity epidemics in highly developed countries.

Adiponectin is a protein generated mainly by white fat tissue, which occurs in 3 isoforms in the serum (LMW low molecular weight, MMW - middle molecular weight, HMW - high molecular weight). The HMW fraction is mainly responsible for the biological activity of adiponectin [11]. Studies show that adiponectin concentration is negatively correlated with BMI, insulin resistance, and fat tissue content [12]. Protective role of adiponectin in the course of metabolic and cardiovascular diseases was confirmed in numerous reports. Adiponectin plays a significant role in glucose metabolism, for instance, through decreasing gluconeogenesis and increasing insulin sensitivity of peripheral tissues. Moreover, it has a vasoprotective effect, stimulating generation of nitric oxide, inhibiting the effect of proinflammatory cytokines and adhesive molecules as well as reducing proliferation of endothelial cells and smooth muscle cells. Additionally, adiponectin presents an antiatherogenic effect, protecting all stages of atherosclerosis development.

The anti-inflammatory effect of adiponectin in the course of metabolic diseases is characterized by complex and multidirectional nature. Clinical studies have shown the suppressive effect of adiponectin on IL- 6 and TNF- $\alpha$ secretion, through macrophages, upon prior stimulation with the lipopolysaccharide vaccine (LPS) as well as the effect of IL-10 increasing secretion and antagonists of IL-1 receptors through dendritic cells and monocytes [13, 14]. On the other hand, studies conducted on the group of obese and diabetic patients demonstrated that TNF- $\alpha$ and IL- 6 have a suppressive effect on the production and multimerization of adiponectin, which indicates the presence of negative feedback between adiponectin and proinflammatory cytokines [15-18]. CSU is characterized by the presence of a chronic inflammatory condition, expressed

Table 1. Adiponectin concentration, body mass index (BMI), and urticaria activity score (UAS) values in studied groups and subgroups

\begin{tabular}{|c|c|c|c|c|c|}
\hline Feature & Tot $\mathbf{U}$ & $\mathbf{U}$ & U-Ae & Control & $p$ \\
\hline $\begin{array}{l}\text { Adiponectin, ng/ml } \\
\text { Median (range) }\end{array}$ & $83.5(29.0-236.0)$ & $82.5(29.0-159.0)$ & $85.5(40.0-236.0)$ & $82.0(29.0-154.0)$ & Ns \\
\hline $\begin{array}{l}\text { BMI } \\
\text { Median (range) }\end{array}$ & $25.8(18.1-30.6)$ & $26.1(18.1-30.6)$ & $25.4(18.9-30.2)$ & $24.8(19.0-29.9)$ & Ns \\
\hline $\begin{array}{l}\text { UAS } \\
\text { Median (range) }\end{array}$ & & $18(10-32)$ & $20(8-28)$ & & Ns \\
\hline
\end{tabular}

$\overline{T o t} U$ - total patient group, $U$ - patients with wheals only, $U$-Ae - patients with wheals and angioedema, Control-control group, $p-$ statistical significance, Ns - statistically non-significant 


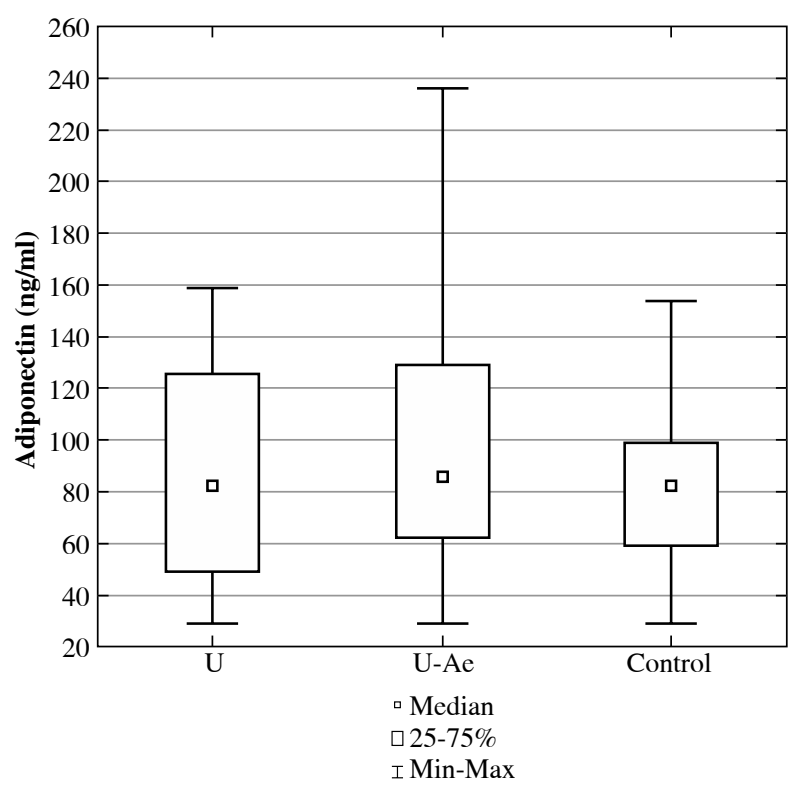

Fig. 1. Adiponectin concentration in studied subgroups (U - patients with wheals only, U-Ae - patients with wheals and angioedema, Control - control group)

by increased concentration of circulating proinflammatory factors such as IL-6, TNF- $\alpha$, IL-1 $\beta$, and CRP [4]. Irrespective of released preformed mediators, the activated mast cells produce a series of cytokines including Il-6, TNF- $\alpha$, VEGF, or PAF, which may affect the level of adiponectin concentration in the blood [19]. The fact that the level of adiponectin in our study did not diverge from the one observed in the control group suggests the complexity of mechanisms regulating adiponectin concentration, including those independent from the course of CSU.

Additionally, a potential role in the CSU pathogenesis is assigned to an excessive activation of coagulation system. In animal models, it was observed that thrombin may induce degranulation of mast cells and increases permeability of blood vessels. Moreover, high concentration of D-dimers and FDP correlates with disease severity [20-22]. Adiponectin is characterized by anticoagulant effect; its concentration is negatively correlated with the fibrinogen and PAI-1 levels [23].

In CSU pathogenesis, some authors indicated an important role of oxidative stress. Rahu et al. observed increased activity of superoxide dismutase, glutathione, and malondialdehyde within urticaria eruptions in CSU patients comparing with control group, with no difference in their activity within the area of skin without lesions [24]. In a study on a pediatric population, Dilek detected increased level of total oxidant status of plasma in CSU patients, when compared with control group [25]. However, other authors did not confirm this correlation [26]. One of the characteristics of adiponectin is its anti-oxidant effect. The studies showed that adiponectin concentration in blood is negatively correlated with the level of oxidative stress markers [27, 28].

Studies of recent years, indicating the proinflammatory effect of adiponectin in the course of autoimmunological diseases stand in opposition to the protective role of adiponectin in the development of obesity-related diseases. Increased adiponectin concentration was observed in patients with type 1 diabetes, rheumatoid arthritis, systemic lupus erythematosus, or inflammatory bowel diseases $[29,30]$. Hence, studies on the role of adiponectin in CSU pathogenesis seem to be of particular importance. Immunological system dysfunctions, including autoimmunological processes, constitute the most commonly occurring reason of incorrect activation and degranulation of mast cells in CSU. Comparability of adiponectin concentration in blood observed in the groups under study may result from heterogeneous character of the factors underlying the disease. According to literature, antibodies directed against $\alpha$ subunit of receptor with high connection with the Fc fragment of IgE antibody (anti-FceRI $\alpha$ ) and anti-IgE antibodies are present only in $30-50 \%$ patients with CSU. In other patients, the share of other mechanisms responsible for mast cell degranulation is considered, including the effect of pseudoallergens or chronic inflammatory process.

The anti-inflammatory and proinflammatory effect of adiponectin depends on the proportion of its isoforms in the blood. HMW adiponectin has mainly proinflammatory effect through inducing the production of IL-6 by monocytes [31]. In our study, we did not analyze the differences in the concentrations of various adiponectin isoforms in the tested groups. It requires further studies in the future. Despite the fact that no statistically important differences was determined in adiponectin concentrations in the blood of population under study, the difference may concern the activity of individual isoforms in the blood between the groups.

So far, only Trinh et al. attempted to analyze the correlation between the adipokines concentration in the blood and fat tissue and CSU development. They analyzed 191 patients with diagnosed CSU, in whom significantly reduced adiponectin concentrations in blood was found, comparing with the control group. Moreover, no correlation between adiponectin concentration and CSU resistance to treatment was observed [32].

\section{Conclusions}

In spite of continuously expanded knowledge, CSU etiopathogenesis has not been completely discovered. At the present stage, individual studies relating to the correlation between adipokines concentration and CSU occurrence do not permit to draw unambiguous conclusions about the role they play in CSU pathogenesis. It is necessary to conduct further studies to comprehensively verify and explain the correlation. 


\section{Acknowledgments}

The study was supported by the grant from the Medical University of Silesia (KNW-1-067/N/5/0).

The authors declare no conflict of interest.

\section{References}

1. Zuberbier T, Aberer W, Asero R, et al. (2014): The EAACI/ GA2LEN/EDF/WAO Guideline for the definition, classification, diagnosis, and management of urticaria: The 2013 revision and update. Allergy 69: 868-887.

2. Zuberbier T, Maurer M (2007): Urticaria: Current Opinions about Etiology, Diagnosis and Therapy. Acta Derm Venereol 87: 196-205.

3. Kasperska-Zajac A (2012): Acute-phase response in chronic urticaria. J Eur Acad Dermatol Venereol 26: 665-672.

4. Dos Santos JC, Azor MH, Nojima VY, et al. (2008): Increased circulating pro-inflammatory cytokines and imbalanced regulatory T-cell cytokines production in chronic idiopathic urticaria. Int Immunopharmacol 8: 1433-1440.

5. Esfahani M, Movahedian A, Baranchi M, Goodarzi MT (2015): Adiponectin: an adipokine with protective features against metabolic syndrome. Iran J Basic Med Sci 18: 430-442.

6. Toussirot E, Binda D, Gueugnon C, Dumoulin G (2012): Adiponectin in autoimmune diseases. Curr Med Chem 19: 5474-5480.

7. Gremese E, Tolusso B, Gigante MR, Ferraccioli G (2014): Obesity as a risk and severity factor in rheumatic diseases (autoimmune chronic inflammatory diseases). Front Immunol 5: 1-10.

8. Lapi F, Cassano N, Pegoraro V, et al. (2016): Epidemiology of chronic spontaneous urticaria: results from a nationwide, population-based study in Italy. Br J Dermatol 174: 996-1004.

9. Zbiciak-Nylec M, Wcisło-Dziadecka D, Kasprzyk M, et al. (2018): Overweight and obesity may play a role in the pathogenesis of chronic spontaneous urticaria. Clin Exp Dermatol 43: 525-528.

10. Ye Y-M, Jin H-J, Hwang E-K, et al. (2013): Co-existence of Chronic Urticaria and Metabolic Syndrome: Clinical Implications. Acta Derm Venereol 93: 156-160.

11. Pajvani UB, Du X, Combs TP, et al. (2003): Structure-function studies of the adipocyte-secreted hormone Acrp30/adiponectin: Implications for metabolic regulation and bioactivity. J Biol Chem 278: 9073-9085.

12. Matsubara M, Maruoka S, Katayose S (2002): Inverse relationship between plasma adiponectin and leptin concentrations in normal-weight and obese women. Eur J Endocrinol 147: 173-80.

13. Weigert J, Neumeier M, Schäffler A, et al. (2005): The adiponectin paralog CORS-26 has anti-inflammatory properties and is produced by human monocytic cells. FEBS Lett 579: $5565-5570$

14. Wolf AM, Wolf D, Rumpold H, et al. (2004): Adiponectin induces the anti-inflammatory cytokines IL-10 and IL-1RA in human leukocytes. Biochem Biophys Res Commun 323: 630-635.

15. Fantuzzi G (2008): Adiponectin and inflammation : consensus and controversy. J Allergy Clin Immunol 121: 326-330.
16. Kern PA, Di Gregorio GB, Lu T, et al. (2003): Adiponectin expression from human adipose tissue: relation to obesity, insulin resistance, and tumor necrosis factor-alpha expression. Diabetes 52: 1779-1785.

17. Lim JY, Kim WH, Park SI (2008): G06976 prevents TNF- $\alpha$-induced suppression of adiponectin expression in 3T3-L1 adipocytes: Putative involvement of protein kinase C. FEBS Lett 582: 3473-3478.

18. He Y, Lu L, Wei X, et al. (2016): The multimerization and secretion of adiponectin are regulated by TNF-alpha. Endocrine 51: 456-68.

19. Jain S (2014): Pathogenesis of Chronic Urticaria: An Overview. Dermatol Res Pract 2014: 674709.

20. Takahagi S, Mihara S, Iwamoto K, et al. (2010): Coagulation/ fibrinolysis and inflammation markers are associated with disease activity in patients with chronic urticaria. Allergy 65 : 649-656.

21. Asero R, Tedeschi A, Marzano AV, Cugno M (2016): Chronic spontaneous urticaria : immune system, blood coagulation, and more. Expert Rev Clin Immunol 12: 229-231.

22. Asero R, Tedeschi A, Riboldi P, Cugno M (2006): Plasma of patients with chronic urticaria shows signs of thrombin generation, and its intradermal injection causes wheal-andflare reactions much more frequently than autologous serum. J Allergy Clin Immunol 117: 1113-1117.

23. Csongrádi É, Káplár M, Nagy B Jr, et al. (2017): Adipokines as atherothrombotic risk factors in obese subjects: associations with haemostatic markers and common carotid wall thickness. Nutr Metab Cardiovasc Dis 27: 571-580.

24. Raho G, Cassano N, D'Argento V, et al. (2003): Over-expression of Mn-superoxide dismutase as a marker of oxidative stress in lesional skin of chronic idiopathic urticaria. Clin Exp Dermatol 28: 318-320.

25. Dilek F, Ozceker D, Ozkaya E, et al. (2016): Oxidative Stress in Children with Chronic Spontaneous Urticaria. Oxid Med Cell Longev 2016: 3831071.

26. Kasperska-Zajac A, Brzoza Z, Polaniak R, et al. (2007): Markers of antioxidant defence system and lipid peroxidation in peripheral blood of female patients with chronic idiopathic urticaria. Arch Dermatol Res 298: 499-503.

27. Furukawa S, Fujita T, Shumabukuro M, et al. (2004): Increased oxidative stress in obesity and its impact on metabolic syndrome. J Clin Invest 114: 1752-1761.

28. Gradinaru D, Margina D, Borsa C, et al. (2017): Adiponectin: possible link between metabolic stress and oxidative stress in the elderly. Aging Clin Exp Res 29: 621-629.

29. Otero M, Lago R, Gomez R, et al. (2006): Changes in plasma levels of fat-derived hormones adiponectin, leptin, resistin and visfatin in patients with rheumatoid arthritis. Ann Rheum Dis 65: 1198-1201

30. Li L, Wu LL (2012): Adiponectin and Interleukin-6 in Inflammation-Associated Disease. Vitam Horm 90: 375-395.

31. Neumeier M, Weigert J, Schäffler A, et al. (2006): Different effects of adiponectin isoforms in human monocytic cells. J Leukoc Biol 79: 803-808.

32. Trinh HKT, Pham D Le, Ban G-Y, et al. (2016): Altered Systemic Adipokines in Patients with Chronic Urticaria. Int Arch Allergy Immunol 171: 102-110. 